\section{(2) OPEN ACCESS}

\title{
Efficacy and safety of low dose ticagrelor in patients with acute coronary syndrome: a systematic review and meta-analysis
}

\author{
Qing Chen, ${ }^{1}$ Yuanyuan Zhang, ${ }^{1}$ Zhen Wang, ${ }^{1}$ Shuai Wang, ${ }^{1}$ Hao Zhang, ${ }^{2}$ \\ Yiwen Wang, ${ }^{1}$ Chi Lu, ${ }^{1}$ Haochen Xuan, ${ }^{3}$ Chaofan Wang, ${ }^{3}$ Dongye Li, ${ }^{1}$ Tongda Xu (ib) 1,3
}

- Additional material is published online only. To view please visit the journal online (http://dx.doi.org/10.1136/ postgradmedj-2019-137180).

${ }^{1}$ Institute of Cardiovascular Disease Research, Xuzhou Medical University, Xuzhou, Jiangsu, China

${ }^{2}$ Department of Thoracic Cardiovascular Surgery, The Affiliated Hospital of Xuzhou Medical University, XuZhou, JiangSu, China

${ }^{3}$ Department of Cardiology, The Affiliated Hospital of Xuzhou Medical University, Xuzhou, Jiangsu, China

\section{Correspondence to}

Dr Tongda Xu, Department of Cardiology, The Affiliated Hospital of Xuzhou Medical University, Xuzhou, Jiangsu, China; xutongda3004@163. com and Dr Dongye Li, Institute of Cardiovascular Disease Research, Xuzhou Medical University, Xuzhou, Jiangsu, China; dongyeli@xzhmu.edu.cn

QC and YZ contributed equally.

Received 28 September 2019 Revised 9 May 2020 Accepted 15 May 2020

\section{ABSTRACT}

Our aim was to examine clinical trials, provide guidance to practitioners and estimate the efficacy and safety of two agents by comparing low dose ticagrelor with standard dose clopidogrel in patients with acute coronary syndrome. We systematically looked through Pubmed, Embase, the Cochrane Library, Wanfang data and CNKI for trials comparing low dose ticagrelor with standard dose clopidogrel for the treatment of patients with ACS since the database was created. The primary endpoint for efficacy was the rate of major adverse cardiac events (MACEs). The primary endpoint for safety was the rate of major bleeding events. We also evaluated platelet function between low dose ticagrelor and standard dose clopidogrel in ACS patients. From 6744 articles, 16 studies including 1629 patients met the inclusion criteria. In contrast with standard dose clopidogrel, low dose ticagrelor significantly reduced MACEs (OR $0.39,95 \% \mathrm{Cl} 0.26,0.58)$ and the difference was statistically significant $(p<0.01)$. No difference was noted for major bleeding events (OR 1.16, $95 \% \mathrm{Cl} 0.43,3.08)$ between the two agents ( $p=0.77)$. In addition, low dose ticagrelor showed lower platelet aggregation rate than clopidogrel (standardised mean difference (SMD) $-0.68,95 \% \mathrm{Cl}-0.83$ to 0.53$)(p<0.01$ ). Platelet reaction units for low dose ticagrelor were much lower than those for standard dose clopidogrel (SMD $-2.46,95 \% \mathrm{Cl}-2.85$ to -2.07$)(\mathrm{p}<0.01)$. In comparison with standard dose clopidogrel, low dose ticagrelor significantly lowered the incidence of MACEs, improved left ventricular ejection fraction, decreased left ventricular end diastolic dimension and did not expand the risk of major bleeding events or minor or minimal bleeding events in ACS patients with a considerable safety and efficacy profile. In addition, low dose ticagrelor was associated with dramatically lower platelet aggregation compared with standard dose clopidogrel.

\section{INTRODUCTION}

Acute coronary syndrome (ACS) is a common cardiovascular disease caused by coronary atherosclerotic stenosis or obstruction, which leads to myocardial ischaemia, hypoxia or myocardial necrosis, threatening human health. It has a high incidence and mortality rate, which seriously affects lifestyle and the prognosis of patients. ${ }^{12}$ Dual antiplatelet therapy (DAPT) as an effective treatment for ACS patients can reduce platelet reactivity and prevent ischaemic events. $^{3-5}$
The current guidelines recommend ticagrelor $90 \mathrm{mg}$ twice daily or clopidogrel $75 \mathrm{mg}$ once daily with aspirin in ACS patients for DAPT usage. These recommendations are founded on large, multicentre, double blind, randomised clinical trials. Compared with clopidogrel, the higher titre P2Y12 inhibitor ticagrelor reduces the incidence of cardiovascular recurrent events in ACS patients after percutaneous coronary intervention (PCI), but in clinical practice, especially in Asia, the risk of bleeding is increased, limiting drug use. ${ }^{6}$

In a controversial study, routine dose ticagrelor was found to increase the risk of major and minor bleeding in ACS patients compared with clopidogrel. ${ }^{7}$ So far, few studies have documented the efficacy of low dose of ticagrelor in ACS patients. Therefore, we assessed the efficacy and safety of low dose ticagrelor compared with standard dose clopidogrel in ACS patients by meta-analysis.

\section{METHODS}

\section{Data extract and search protocol}

This meta-analysis was conducted according to the Preferred Reporting Items for Systematic Reviews and Meta-Analyses (PRISMA) statement. ${ }^{8}$ The protocol of this meta-analysis was registered in advance by the International Prospective Register of Systematic Reviews (PROSPERO, identification No CRD42020171621). Our group found relevant publications comparing low dose ticagrelor with standard dose clopidogrel for the treatment of patients with ACS, with no language restrictions, in the databases Pubmed, Embase, Cochrane Library, Wanfang data and CNKI. The following search terms or phrases were used: 'ticagrelor, clopidogrel'. The terms 'coronary artery disease' and 'acute coronary syndrome' were also included in the search strategy (see online supplementary data S1 for full list). In addition, we also hand searched the official websites of highly qualified journals (eg, New England Journal of Medicine, The Lancet, Journal of the Circulation and American College of Cardiology) for relevant documents. We took all potentially eligible studies for review into account, irrespective of country or language.

\section{Inclusion and exclusion criteria}

Inclusion criteria

1. Clinical trials that compared low dose ticagrelor with standard dose clopidogrel in ACS patients with all forms. 
2. The observation group of the intervention was low dose ticagrelor and the control group was standard dose clopidogrel. The rest of the intervention measures were consistent and the treatment course was not limited.

3. The studies were eligible and provided accurate and complete statistical data, including at least one outcome: major adverse cardiac event (MACEs), major bleeding events, minor or minimal bleeding events, non-bleeding adverse events (AEs), left ventricular ejection fraction (LVEF), left ventricular end diastolic dimension (LVDD), platelet aggregation rate (PAgR) and platelet reaction unit (PRU) values.

\section{Exclusion criteria}

1. The research did not compare low dose ticagrelor with standard dose clopidogrel.

2. The data were unsuitable to be used in this meta-analysis.

3. Review articles, clinical trials, case reports and discussion papers.

\section{Primary indices assessing efficacy}

- MACEs: cardiovascular death, myocardial infarction and stroke. ${ }^{9}$

- LVEF.

- LVDD.

\section{Secondary indices assessing safety}

- Major bleeding events: fatal bleeding, intrapericardial bleeding with cardiac tamponade, intracranial bleeding, hypovolaemic shock or severe hypotension due to bleeding and requiring pressors or surgery, clinically overt or apparent bleeding associated with a decrease in haemoglobin of $>5 \mathrm{~g} /$ $\mathrm{dL}$, or transfusion of $\mathrm{C} 4$ units of whole blood or packed red blood cells for bleeding. ${ }^{9} 10$

- Minor bleeding events: medical intervention needed to stop or treat the bleeding when necessary. ${ }^{9-11}$

- Minimal bleeding events: medical intervention is not needed or no treatment is required for the bleeding events. ${ }^{9-11}$

- Non-bleeding adverse events: dyspnoea, bradycardia, diarrhoea, ventricular tachycardia and drug discontinuation, but particularly dyspnoea. ${ }^{9-11}$

\section{Outcomes assessing platelet reactivity}

- Platelet aggregation rate (PAgR) using traditional light transmission aggregometry to assess platelet aggregation. ${ }^{9} 12$

- Platelet reaction units (PRU) using the VerifyNow assay to measure platelet aggregation. ${ }^{9} 12$

\section{Data extraction}

Two independent researchers reviewed the study titles and abstracts, and potentially relevant articles satisfying the inclusion criteria were reviewed in detail. Trials selected for data extraction and detailed analysis were performed by two researchers. Disagreements were resolved by a third researcher.

The bias risk was evaluated according to the modified Jadad scale. A score of 2 means a low risk of bias, a score of 1 implies unclear bias risk or medium risk, and a score of 0 represents a high risk of bias. Scores were given to each of the four components.

\section{Method of meta-analysis and evaluation of heterogeneity reported bias}

For the dichotomous outcome variables (including MACEs, major bleeding events, minimal bleeding events and non- bleeding adverse events) the Peto method was used to weight the statistics. We found that it showed better statistical properties when there were few events or the data were sparse, regardless of the low event rates or small study size. For continuous outcome variables (including LVEF, LVDD, PAgR and PRU) the inverse variance method was used to weight the statistics. ${ }^{13}$

Heterogeneity was evaluated by two statistical techniques: the Cochrane $\mathrm{Q}$ statistic test and the $\mathrm{I}^{2}$ statistic test. A p value $<0.05$ or $\mathrm{I}^{2}>50 \%$ indicated high heterogeneity. Also, a narrower CI means the reliability of the estimation of the population mean from the sample mean was better. The point estimate around $50 \%$ also indicates better reliability of the estimation of the population mean from the sample mean. A fixed effects model was used to combine the data when $\mathrm{I}^{2} \leq 50 \%$, and a random effects model was performed when $\mathrm{I}^{2}$ was $>50 \%$. In the metaanalysis, a fixed effects model was used to combine the data.

In the meta-analyses for each outcome, a funnel plot was constructed with each trial's effect size and SE, to assess publication bias. Begg's test and Egger's test were used to evaluate funnel plot asymmetry, and significant publication bias was defined as $\mathrm{p}<0.1$. We used the trim-and-fill computation to evaluate the effect of publication bias on the interpretation of the results, and calculated odds ratios (OR) and 95\% CIs with Stata (V.11.0).

\section{RESULTS}

\section{Searched outcomes}

We researched the literature on low dose ticagrelor before starting this meta-analysis. We considered ticagrelor $90 \mathrm{mg}$ once a day, $45 \mathrm{mg}$ twice a day and $60 \mathrm{mg}$ twice a day as low dose ticagrelor, based on extracted data from five studies. ${ }^{14-18}$ A total of 6744 articles were selected through database searching. We assessed the abstracts of these articles and 62 potentially relevant articles were reviewed in detail. Consequently, 16 publications underwent qualitative and quantitative analysis (figure 1).

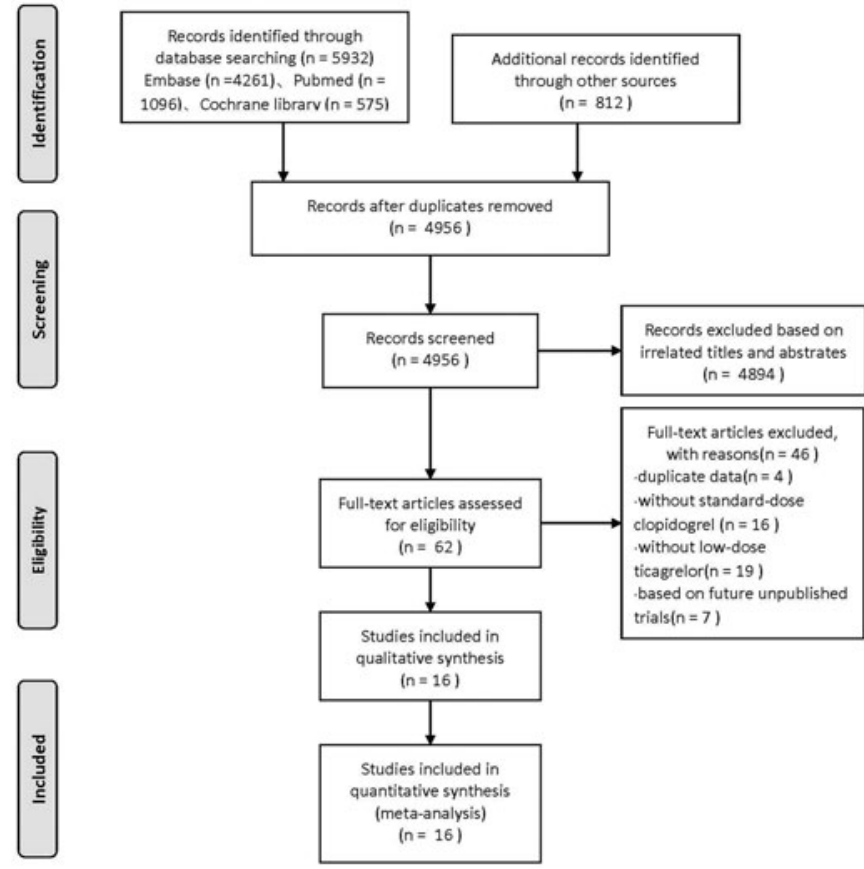

Figure 1 Preferred Reporting Items for Systematic Reviews and MetaAnalyses (PRISMA) flow diagram showing the study selection process. 


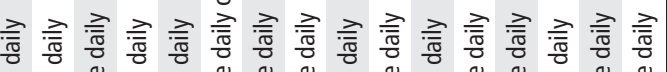

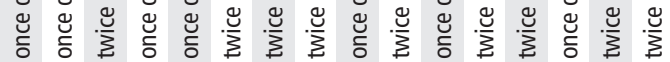

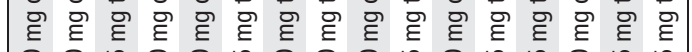

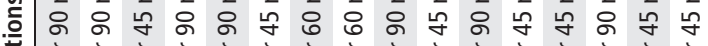

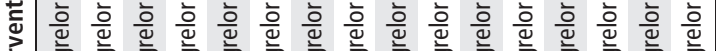

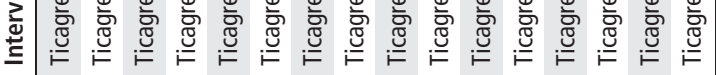
$\stackrel{\mathscr{n}}{\underline{\underline{*}}}$

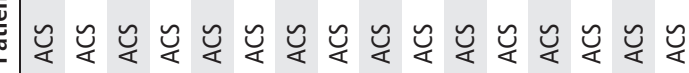

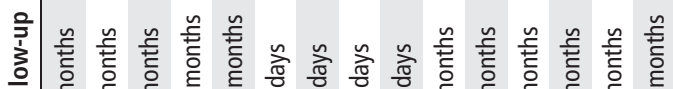

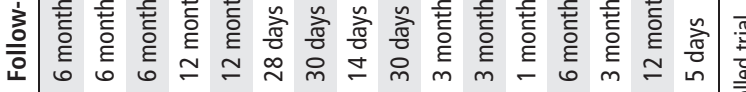
@

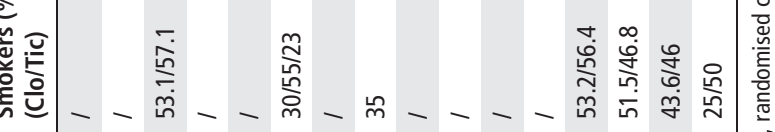

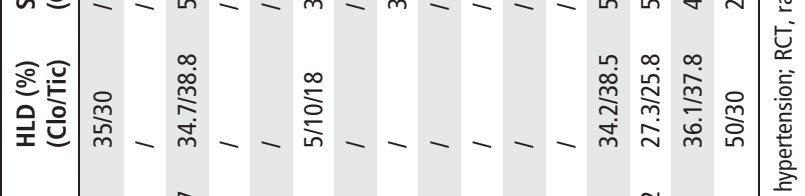

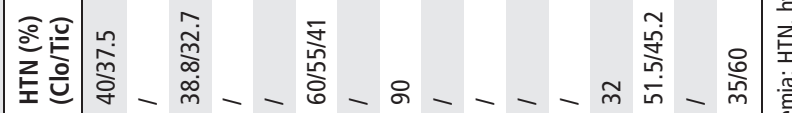

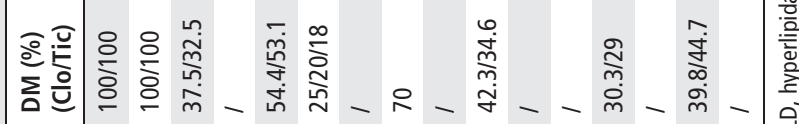

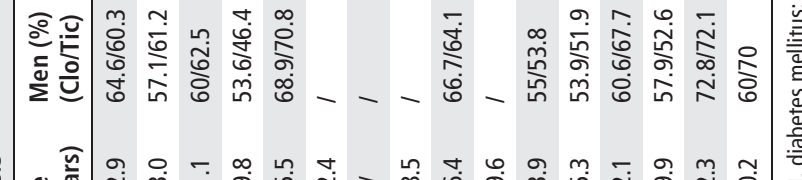

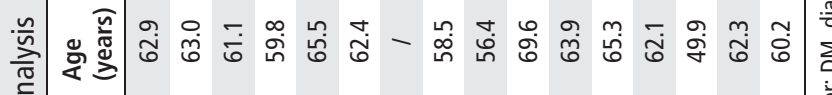
垔

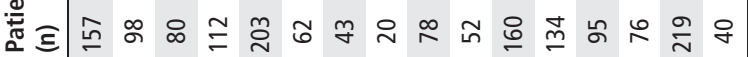
은

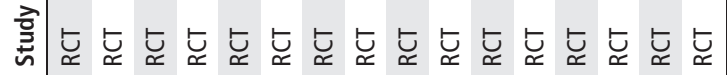

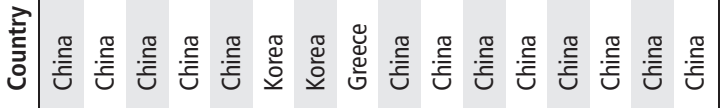



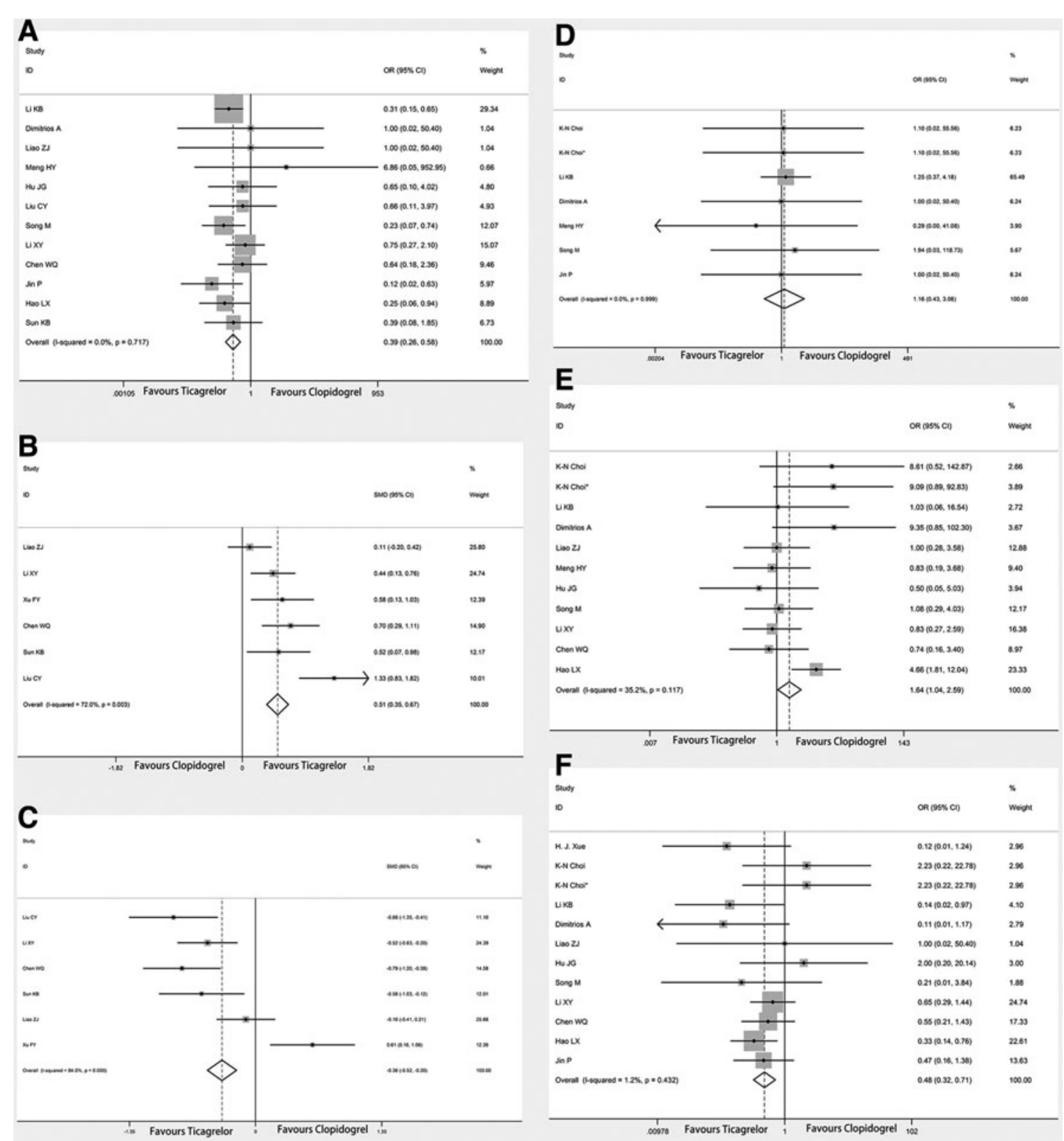

Figure 2 Forest plot showing efficacy (primary outcomes) and safety (secondary outcomes) observed between low dose ticagrelor and standard dose clopidogrel, including (A) MACEs, (B) LVEF, (C) LVDD, (D) major bleeding events, (E) minor or minimal bleeding events and (F) AEs. MACEs, major adverse cardiac events; LVDD, left ventricular end diastolic dimension; LVEF, left ventricular ejection fraction; AEs, non-bleeding adverse events.

\section{Description of studies}

Sixteen studies with 1629 patients, 756 patients treated with low dose ticagrelor and 873 patients treated with standard dose clopidogrel, were recruited in this analysis. ${ }^{3-7} 91112$ 19-25 Some differences existed in the included studies regarding study design and patient characteristics. All studies were randomised controlled trials and published in 2015-2018. Patients were from territories worldwide, but especially from Korea, Greece and China. However, the doses of ticagrelor used in these studies were different, including 484 patients who received ticagrelor $90 \mathrm{mg}$ once a day, ${ }^{34679192124} 240$ patients who received ticagrelor 45 mg twice a day 52022232526 and 32 patients who received ticagrelor $60 \mathrm{mg}$ twice a day. ${ }^{11}{ }^{12}$ All participants received aspirin $75-125 \mathrm{mg}$ and did not receive other antithrombotic agents. However, non-antithrombotic agents were used, including beta receptor antagonists, statins and angiotensin converting enzyme inhibitors, among others. Individual study characteristics, patient characteristics and inclusion/exclusion criteria of the included studies are shown in table 1.

\section{Primary outcomes (outcomes representing efficacy)}

Compared with standard dose clopidogrel, low dose ticagrelor significantly reduced MACEs (OR 0.39, 95\% CI 0.26, 0.58), ${ }^{3} 46$ 712 19-25 increased LVEF (standardised mean difference (SMD) $0.51,95 \%$ CI $0.35,1.82)^{3-5} 192124$ and decreased LVDD (SMD $-0.36,95 \% \mathrm{CI}-0.52$ to -0.20$),{ }^{3419} 24$ and the differences were significant $(\mathrm{p}<0.01)$ (figure 2$)$. However, the $\mathrm{I}^{2}$ value for studies assessing changes in LVEF was $72.0 \%$ in ACS patients, indicating significant heterogeneity across the studies. Subgrouping according to age, region, follow-up duration and dose of ticagrelor had no pronounced effect on the $\mathrm{I}^{2}$ values, but $\mathrm{I}^{2}$ was reduced to $37.5 \%$ after excluding data for one study with higher weightings $(>50 \%)$. Similarly, the $\mathrm{I}^{2}$ value for studies assessing changes in LVDD was $84.0 \%$, and $\mathrm{I}^{2}$ was reduced to 


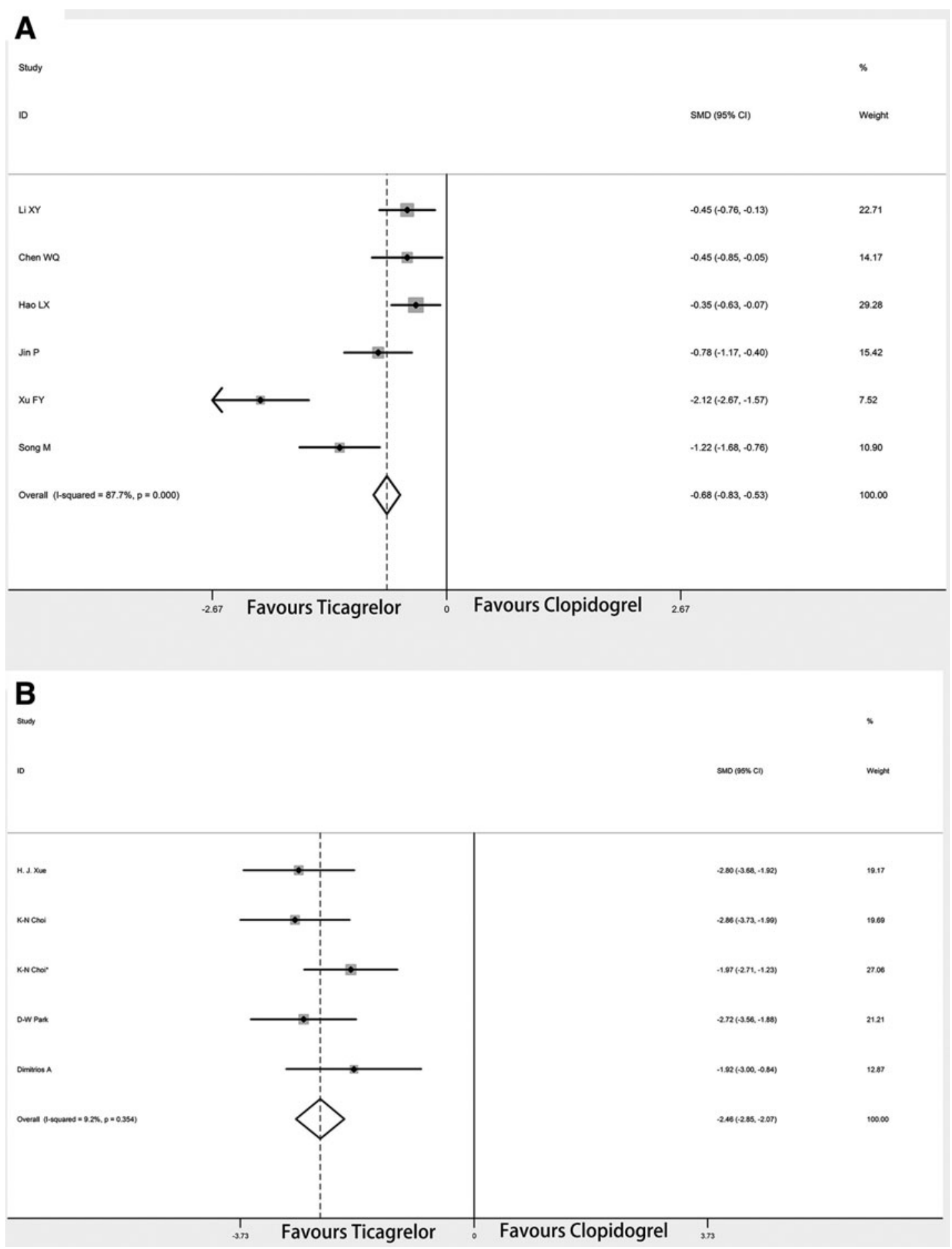

Figure 3 Forest plot showing platelet reactivity observed between low dose ticagrelor and standard dose clopidogrel, including (A) platelet aggregation rate and (B) P2Y12 reaction units.

0 after excluding data from two studies with higher weightings $(>50 \%)$.

\section{Secondary outcomes (outcomes representing safety)}

The $\mathrm{I}^{2}$ value for studies assessing safety outcomes was $\leq 50 \%$ in ACS patients, and a fixed effects model was used to combine the data. Major bleeding events were not significantly different with low dose ticagrelor versus standard dose clopidogrel (OR 1.16, 95\% CI 0.43, 3.08; $\mathrm{p}=0.77$ ). ${ }^{6912222325}$ Compared with standard dose clopidogrel, low dose ticagrelor significantly decreased the incidence of minor or minimal bleeding events (OR 1.64, 95\% CI 1.06, 2.59; $\mathrm{p}=0.04)^{3} 46791220-2325$ and AEs (OR 0.48, 95\% CI 0.32, 0.71; $\mathrm{p}<0.01)^{3} 479122021232526$ (figure 2).

\section{Outcomes representing platelet reactivity}

Low dose ticagrelor showed lower PAgR than clopidogrel (SMD $-0.68,95 \%$ CI -0.83 to $-0.53 ; \mathrm{p}<0.01) .{ }^{346723}$ PRU values for low dose ticagrelor were also lower than those for standard dose clopidogrel (SMD $-2.46,95 \%$ CI -2.85 to -2.07 ; $\mathrm{p}<0.01$ ) (figure 3). ${ }^{911} 1226$ 
Table 2 Univariate meta-regression for efficacy and safety of low dose ticagrelor in patients with acute coronary syndrome

\begin{tabular}{|c|c|c|c|c|c|c|c|c|}
\hline Covariate & MACE & LVEF & LVDD & $\begin{array}{l}\text { Major bleeding } \\
\text { events }\end{array}$ & $\begin{array}{l}\text { Minor or minimal bleeding } \\
\text { events }\end{array}$ & $\begin{array}{l}\text { Non-bleeding } \\
\text { adverse events }\end{array}$ & PAgR & PRU \\
\hline $\begin{array}{l}\text { Average } \\
\text { age }\end{array}$ & $\begin{array}{l}1.01(0.47,2.18)^{*} \\
(p=0.97)\end{array}$ & $\begin{array}{l}1.60(0.66,3.84) \\
R^{2}=29.05 \% \\
(p=0.21)\end{array}$ & $\begin{array}{l}0.59(0.16,2.18) R^{2}=4.94 \%, \\
(p=0.33)\end{array}$ & $\begin{array}{l}0.91(0.14,5.94)^{*} \\
(p=0.91)\end{array}$ & $\begin{array}{l}2.34(0.80,6.81) \\
R^{2}=100.00 \%,(p=0.11)\end{array}$ & $\begin{array}{l}0.75(0.39,1.45)^{*} \\
(p=0.36)\end{array}$ & $\begin{array}{l}1.23(0.42,3.58) \\
R^{2}=-20.93 \% \\
(p=0.62)\end{array}$ & $\begin{array}{l}1.46(0.46,4.62) \\
R^{2}=1.06 \%, \\
(p=0.37)\end{array}$ \\
\hline Region & $\begin{array}{l}2.48(0.25,246.59)^{*} \\
(p=0.67)\end{array}$ & NR & NR & $\begin{array}{l}0.91(0.14,6.09)^{*} \\
(p=0.91)\end{array}$ & $\begin{array}{l}2.79(0.77,10.10) \\
R^{2}=15.01 \%,(p=0.10)\end{array}$ & $\begin{array}{l}1.90(0.70,5.15)^{*} \\
(p=0.18)\end{array}$ & NR & $\begin{array}{l}1.09(0.42,2.79) \\
R^{2}=-192.02 \%, \\
(p=0.80)\end{array}$ \\
\hline Follow-up & $\begin{array}{l}0.58(0.18,1.81)^{*} \\
(p=0.31)\end{array}$ & $\begin{array}{l}0.96(0.35,2.61) \\
R^{2}=-37.57 \% \\
(p=0.91)\end{array}$ & $\begin{array}{l}1.30(0.34,4.97) \\
R^{2}=-21.93 \%,(p=0.61)\end{array}$ & $\begin{array}{l}1.10(0.06,19.13)^{*} \\
(p=0.94)\end{array}$ & $\begin{array}{l}0.94(0.22,4.11) \\
R^{2}=-42.63 \%,(p=0.93)\end{array}$ & $\begin{array}{l}0.50(0.12,2.04)^{*} \\
(p=0.30)\end{array}$ & $\begin{array}{l}0.85(0.14,5.07) \\
R^{2}=-28.48 \% \\
(p=0.82)\end{array}$ & NR \\
\hline $\begin{array}{l}\text { Ticagrelor } \\
\text { dose }\end{array}$ & $\begin{array}{l}1.28(0.75,2.20)^{*} \\
(p=0.33)\end{array}$ & $\begin{array}{l}1.01(0.26,3.99) \\
R^{2}=35.32 \% \\
(p=0.98)\end{array}$ & $\begin{array}{l}0.32(0.11,0.87) \\
R^{2}=75.25 \%,(p=0.03)\end{array}$ & $\begin{array}{l}0.89(0.13,5.90)^{*} \\
(p=0.88)\end{array}$ & $\begin{array}{l}1.22(0.58,2.55) \\
R^{2}=-22.28 \%,(p=0.56)\end{array}$ & $\begin{array}{l}0.98(0.47,2.05)^{*} \\
(p=0.94)\end{array}$ & $\begin{array}{l}3.09(1.32,7.20) \\
R^{2}=87.07 \% \\
(p=0.02)\end{array}$ & $\begin{array}{l}1.54(0.70,3.36) \\
R^{2}=100 \% \\
(p=0.18)\end{array}$ \\
\hline Language & $\begin{array}{l}2.48(0.03,246.59)^{*} \\
(p=0.67)\end{array}$ & NR & NR & $\begin{array}{l}0.84(0.03,23.08)^{*} \\
(p=0.90)\end{array}$ & $\begin{array}{l}6.73(0.72,63.22) \\
R^{2}=17.11 \%,(p=0.07)\end{array}$ & $\begin{array}{l}1.44(0.28,7.43)^{*} \\
(p=0.63)\end{array}$ & NR & NR \\
\hline
\end{tabular}

${ }^{*}$ Adj R-squared $=0 \%$

LVDD, left ventricular end diastolic dimension; LVEF, left ventricular ejection fraction; MACE, major adverse cardiac event; NR, not reported; PAgR, platelet aggregation rate; PRU, platelet reaction units.

\section{Heterogeneity analyses}

Heterogeneity in the full meta-analysis without population stratification for the between study variance and the within study variance was shown. Therefore, meta-regression and subgroup analysis was required to explain the heterogeneity. From the results of the full meta-analysis, higher heterogeneity was found for LVEF, LVDD and PAgR, and the $\mathrm{I}^{2}$ values were $72 \%, 84 \%$ and $87.7 \%$, respectively. Therefore, we performed a meta-regression to explore the source of heterogeneity (tables 2 and 3, online supple mentary figure S1). In accordance with data availability of the studies, factors analysed were: average age ( $\leq 60$ years, $>60$ and $\leq 65$ years, $>65$ years); region (China, Korea, Greece); follow-up (intervention effect $\leq 3$ months, intervention effect $\geq 6$ months); ticagrelor dose (45 mg twice daily, $60 \mathrm{mg}$ twice daily, $90 \mathrm{mg}$ once daily); and language (English, Chinese). We found that the dose of ticagrelor contributed a lot to the source of heterogeneity of LVDD and PAgR, and the corrected $\mathrm{R}^{2}$ values were $75.25 \%$ and $87.07 \%$ $(p=0.03$ and $p=0.02)$. No significant factors were observed for heterogeneity of LVEF in the meta-regression. There may be other potential factors causing heterogeneity. Heterogeneity in each subgroup was displayed by subgroup meta-analysis, and the results are shown in table 4 and online supplementary file 1 . Age $<65$ years, Chinese studies and long term follow-up ( $\geq 6$ months) were associated with greater efficacy and safety of low dose ticagrelor in ACS patients. A difference was found between the two doses of ticagrelor for PAgR (SMD -0.66, 95\% CI -0.83 to -0.53 ; $\mathrm{p}<0.01$ ), the $\mathrm{I}^{2}$ statistic was reduced from $87.7 \%$ to $7.8 \%$, without altering the significance of the pooled effect size, when studies were restricted to those in which patients received ticagrelor $45 \mathrm{mg}$ twice a day. A small sample size for each dose of ticagrelor in the studies may also be responsible for the heterogeneity of the results. This analysis included Chinese and English studies, and subgroup analysis showed that language differences did not affect the overall results. Gablbraith plot and Metaninf plot were also made to estimate the heterogeneity of the included studies and the impact of individual studies on the combined effect (see online supplementary figure S3 and figure S4).

\section{Study quality and publication bias}

The quality of the randomised studies, as assessed by the modified Jadad scale, ranged from moderate to high quality. with scores of 5-7 (see online supplementary table S1). A funnel plot of each trial's effect size was constructed by Begg's test and Egger's test to assess the possibility of publication bias (online supplementary figure S5, online supplemen tary table S2). No significant publication bias was found in the analysis $(p>0.1)$. No study exceeding the 95\% CI was shown in the funnel plot, and the studies gathered at the top of the funnel chart, which also suggests low selection bias or publication bias in this analysis.

\section{DISCUSSION}

This meta-analysis suggests that low dose ticagrelor could significantly reduce MACEs, increase LVEF and decrease LVDD

Table 3 Adjusted $\mathrm{p}$ value of multi-factor meta-regression analysis for the efficacy and safety of low dose ticagrelor in patients with acute coronary syndrome

\begin{tabular}{|c|c|c|c|c|c|c|c|c|}
\hline Covariate & MACE & LVEF & LVDD & Major bleeding events & Minor or minimal bleeding events & Non-bleeding adverse events & PAgR & PRU \\
\hline Average age & 0.80 & 0.49 & 0.20 & 0.97 & 0.20 & 0.85 & 0.42 & 0.74 \\
\hline Region & 1.00 & NR & NR & 1.00 & 0.83 & 0.22 & NR & 0.98 \\
\hline Follow-up & 0.52 & 0.64 & 0.27 & 0.97 & 0.65 & 0.51 & 0.24 & NR \\
\hline Ticagrelor dose & 0.75 & 1.00 & 0.04 & 1.00 & 0.78 & 0.98 & 0.02 & 0.24 \\
\hline Language & NR & NR & NR & 1.00 & 0.99 & 0.17 & NR & NR \\
\hline
\end{tabular}

Monte Carlo permutation test for meta-regression correction (permutations=10 000).

LVDD, Left ventricular end diastolic dimension; LVEF, Left ventricular ejection fraction; MACEs, Major adverse cardiac events; NR, Not reported; PAgR, platelet aggregation rate; PRU, Platelet reaction units. 


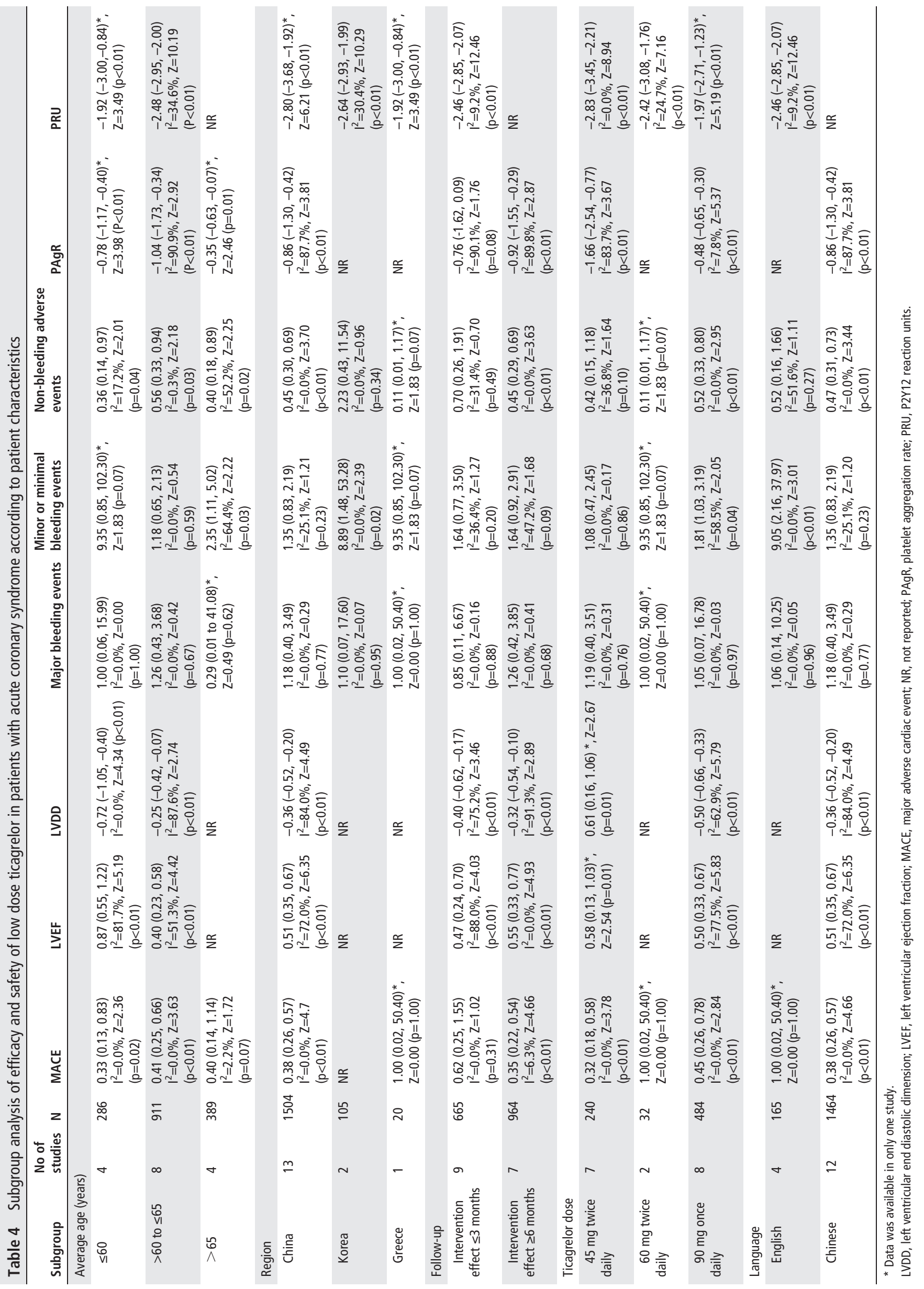


compared with standard dose clopidogrel in ACS patients. No differences for the risk of major bleeding events were shown between the two groups. Less incidence of minor or minimal bleeding events and AEs was observed in the low dose ticagrelor group. In addition, low dose ticagrelor was associated with lower platelet aggregation compared with standard dose clopidogrel.

The platelet inhibition and patient outcomes (PLATO) study, an important clinical study involving 18624 patients from different countries and centres, showed that ticagrelor significantly reduced cardiovascular death, myocardial infarction and stroke events compared with clopidogrel (absolute risk reduction 16\%, relative risk reduction $16 \%, \mathrm{p}<0.001$, numbers needed to treat $=54$ ). Also, the risk of MACEs with ticagrelor decreased in the early stage and was maintained throughout the 1 year treatment. ${ }^{4}$ 27-29 Ticagrelor did not increase major bleeding or fatal/life threatening major bleeding. Ticagrelor related dyspnoea events were higher that those with clopidogrel, but most events were mild to moderate and could be relieved without treatment. However, ticagrelor increased the risk of minor or minimal bleeding events compared with clopidogrel, resulting in a reduction in patient compliance, which could increase the incidence of MACEs.

The PLATO study showed that ticagrelor has great efficacy and safety in ACS patients compared with clopidogrel. However, the PLATO study mainly comprised Western patients, which has limitations. A large number of clinical studies have demonstrated that the clinical efficacy of ticagrelor in East Asian ACS patients is significantly different from that in Western patients. ${ }^{30} 31$ The PHILO (phase the international study of ticagrelor andclinical outcomes in Asian ACS patients) study compared the efficacy and safety of ticagrelor and clopidogrel in ACS patients in East Asia, which was designed in the same way as the PLATO study. In the PHILO study, ticagrelor increased composite events for cardiovascular death, myocardial infarction and stroke compared with clopidogrel (9.0\% vs $6.3 \%, \mathrm{HR}=1.47,95 \%$ CI 0.88 to 2.44$)$. Also, the incidence of bleeding events caused by ticagrelor was higher than that for clopidogrel $(10.3 \%$ vs $6.8 \%, \mathrm{HR}=1.54,95 \%$ CI 0.94 to 2.53 ), which may be related to the low body mass index in East Asian. AEs were more common in the ticagrelor group. ${ }^{32} 33$

The PHILO study showed that ticagrelor does not have an advantage over clopidogrel in ACS patients from East Asia. ${ }^{34}$ Can low doses of ticagrelor reduce adverse events? Our metaanalysis assessed the efficacy and safety of low dose ticagrelor compared with standard dose clopidogrel in ACS patients. In the current analysis, low dose ticagrelor also reduced the incidence of MACEs, did not increase major bleeding events and reduced the incidence of minor or minimal bleeding events and AEs compared with standard dose clopidogrel.

According to the results of this meta-analysis, the different mechanisms of action of ticagrelor and clopidogrel may lead to different outcomes. Clopidogrel, a thienopyridine prodrug, selectively inhibits the binding of ADP to its platelet receptor and secondary ADP mediated activation of the glycoprotein GPllb/llla complex, thereby inhibiting platelet aggregation. ${ }^{1} 35$ Ticagrelor, an oral P2Y12 inhibitor, can bind reversibly to its platelet receptor with a more rapid onset of action and a faster recovery of platelet function. It is preferable to use ticagrelor rather than clopidogrel in ACS patients undergoing an early invasive or ischaemia guided strategy. ${ }^{32-34} 36$

The guidelines recommended that in all ACS patients without contraindications, the ticagrelor protocol should be carried out (180 mg loading dose, then $90 \mathrm{mg}$ twice daily) for up to 12 months. ${ }^{2935}$ If it is not appropriate for some patients to take ticagrelor because of hypersensitivity or major bleeding events, a loading dose of clopidogrel should be taken, and then a daily maintenance dose $(300 \mathrm{mg}$ or $600 \mathrm{mg}$ loading dose, and then 75 mg daily). ${ }^{6} 17$

A recently published review put forward the concept of 'East Asian paradox'. ${ }^{37}$ Compared with Westerners, East Asians have a higher incidence of warfarin related intracranial haemorrhage. Therefore, different a target for the international normalised ratio (INR) has been proposed and has been adjusted in clinical

Main messages

- Acute coronary syndrome (ACS) is a common cardiovascular disease that has a high incidence and mortality rate, which seriously affects the quality of life and life expectancy of patients.

- Dual antiplatelet therapy as an effective treatment for ACS patients can reduce platelet reactivity and prevent ischaemic events, and forms the cornerstone of treatment for patients with ACS.

- It is recognised that ticagrelor significantly reduced cardiovascular death, myocardial infarction and stroke events compared with clopidogrel. Ticagrelor did not increase major bleeding or fatal/life threatening major bleeding. However, the incidence of bleeding events was higher in the ticagrelor group than in the clopidogrel group in East Asian ACS patients, which may be related to issues such as low body mass index and ethnic differences in thrombogenicity in East Asians.

Current research questions

Efficacy and safety of aspirin plus ticagrelor $60 \mathrm{mg}$ long term dual antiplatelet therapy in coronary heart disease patients with high risk factors for ischaemia.

- The significance of cardiac MRI in evaluating viable myocardium in patients with coronary heart disease.

- The risk of bleeding events of rivaroxaban on the basis of antiplatelet therapy in patients with atrial fibrillation after percutaneous coronary intervention.

\section{Key references}

1. Jeong YH. "East Asian paradox": challenge for the current antiplatelet strategy of "one-guideline-fits-all races" in acute coronary syndrome. Curr Cardiol Reports 2014;16:485.

2. PHILO Trial. A randomised, double-blind, double-dummy, parallel group, international, multicenter, phase three study to assess the efficacy and safety of ticagrelor vs clopidogrel on top of low dose acetylsalicylic acid in Asian/Japanese patients with non-ST or ST elevation ACS for whom PCl is planned. Available from: http:// www. astrazenecaclinicaltrials.com/_mshost800325/content/clinical-trials /resources/pdf/D5130C00027.

3. Cannon CP, Harrington RA, James $S$, et al. Comparison of ticagrelor with clopidogrel in patients with a planned invasive strategy for acute coronary syndromes (PLATO): a randomised double-blind study. Lancet 2010;375:283-93.

4. Amsterdam EA, Wenger NK, Brindis RG, et al. AHA/ACC guideline for the management of patients with non-ST-elevation acute coronary syndromes: executive summary: a report of the American College of Cardiology/American Heart Association Task Force on Practice Guidelines. Circulation 2014;130:2354-94. 


\section{Self assessment questions}

1. Dual antiplatelet therapy (DAPT) is the cornerstone of treatment for patients with acute coronary syndrome (ACS).

2. The present guidelines recommend that DAPT should last for 2 years.

3. Compared with clopidogrel, ticagrelor has better effectiveness and reduces the incidence of cardiovascular event recurrence in ACS patients with percutaneous coronary intervention.

4. Ticagrelor is an oral P2Y12 inhibitor bound reversibly to its platelet receptor, has a more rapid onset of action and a faster recovery of platelet function.

5. The plausible explanations for the higher incidence of bleeding events in the ticagrelor group compared with the clopidogrel group in East Asian ACS patients which may be related to low body mass index, ethnic differences in thrombogenicity in East Asian and gene-environment interactions.

practice in East Asia (1.6-2.6). Similarly, a large number of clinical data have indicated that the incidence of ischaemic events in East Asians may be similar or even lower compared with Westerners. In contrast, East Asians seem to have a higher risk of bleeding than Westerners. ${ }^{38} 39$

Differences in the effects of coagulation, fibrinolysis and inflammation markers on thrombogenicity among races may be one of the causes of the East Asian paradox. ${ }^{37} 4041$ One potential mechanism is the difference in genetic polymorphisms among different races. In order to evaluate important variables of the clopidogrel response, we reviewed numerous pharmacodynamic studies and clinical evidence, which found that the cytochrome P450 (CYP) 2C19 loss of function allele was the main indicator of platelet reactivity during treatment with clopidogrel. ${ }^{42-44}$ These hypercoagulable factors also exhibit different clinical characteristics, which are regulated by gene-environment interactions. ${ }^{38} 39$

Li et al conducted a study of $90 \mathrm{mg}$ and $180 \mathrm{mg}$ doses of ticagrelor in healthy people in China. The study showed that the maximum plasma concentration and maximum area of the plasma concentration-time curve for ticagrelor $(90 \mathrm{mg}$ twice per day) and its active metabolite (AR-C124910XX) in healthy Chinese volunteers were usually $40 \%$ higher than that in Caucasians. ${ }^{1045}$ Recent studies showed that low dose ticagrelor may have sufficient inhibitory effect on platelet aggregation in Chinese patients with ACS. Our meta-analysis obtained similar results. Avoiding a loading dose of ticagrelor might prevent bleeding events, which may have important clinical significance in the treatment and management of ACS patients in East Asia.

Our meta-analysis mainly involved East Asian patients. The results showed that low dose ticagrelor decreased MACEs and did not increase the risk of major bleeding in ACS patients compared with standard dose clopidogrel, and the rates of minor or minimal bleeding events and AEs events also decreased. This can greatly improve compliance in patients with ACS in East Asia, which will decrease the incidence of MACEs. Low dose ticagrelor may be more suitable for East Asians.

A few potential limitations may exist in this meta-analysis. Some important confounding factors might affect the final results, such as study design, follow-up time, inclusion and exclusion criteria, and ticagrelor dose. For example, the various definitions of MACEs may obscure possible differences in their components. Most of the included studies were positive, which might result in publication bias. In view of the issues mentioned, the authors' conclusions should be treated with caution.

\section{CONCLUSIONS}

On the basis of this meta-analysis, it could be concluded that low dose ticagrelor has a considerable safety and efficacy profile and decreased MACEs and did not increase the risk of major bleeding in ACS patients compared with standard dose clopidogrel. In addition, low dose ticagrelor had lower platelet aggregation compared with standard dose clopidogrel. Therefore, for clinical efficacy and safety, it is necessary to perform large scale clinical trials to identify the optimal antiplatelet regimen and therapeutic level of platelet reactivity.

Acknowledgements We greatly acknowledge the assistance of the Statistical Department of Xuzhou Medical University.

Contributors TX was responsible for the plan for the current study. TX and QC formulated specific search strategy and looked through Pubmed, Embase, the Cochrane Library and other databases in detail for relevant publications. Three independent researchers (ZW, SW and HZ) reviewed the study titles and abstracts, and potentially relevant articles satisfying the inclusion criteria were reviewed in detail. Trials selected for data extraction and detailed analysis were analysed by two researchers (QC and YW). Disagreements were resolved by a third researcher (CL). QC YZ and CL performed the meta-analysis for the included studies through Stata (V.11.0). HX and CW assisted in interpreting the results. QC and YZ drafted the manuscript. TX and DL evaluated the data quality and approved the final version of the manuscript for submission. All authors have critically reviewed the manuscript.

Funding Key Research Developmental Programme of Jiangsu Province (social development No BE2019639); Jiangsu Province Postgraduate Practice Innovation Program (No SJCX19_0946); and Jiangsu Provincial Administration of Traditional Chinese Medicine (No YB201988).

Competing interests None declared.

\section{Patient consent for publication Not required.}

Provenance and peer review Not commissioned; externally peer reviewed.

Open access This is an open access article distributed in accordance with the Creative Commons Attribution Non Commercial (CC BY-NC 4.0) license, which permits others to distribute, remix, adapt, build upon this work non-commercially, and license their derivative works on different terms, provided the original work is properly cited, appropriate credit is given, any changes made indicated, and the use is noncommercial. See: http://creativecommons.org/licenses/by-nc/4.0/.

\section{ORCID iD}

Tongda Xu http://orcid.org/0000-0002-5594-3992

\section{REFERENCES}

1 Roffi M, Patrono C, Collet J-P, et al. 2015 ESC guidelines for the management of acute coronary syndromes in patients presenting without persistent ST-segment elevation: Task force for the management of acute coronary syndromes in patients presenting without persistent ST-segment elevation of the European Society of Cardiology (ESC) Eur Heart J 2016;37:267-315

2 Roger VL, Go AS, Lloyd-Jones DM, et al. Executive summary: heart disease and stroke statistics--2012 update: a report from the American Heart Association. Circulation 2012;125:188-97.

3 XY L, Dong Q, Chen MS. Myocardial protective effect of ticagrelor on ST-segment elevation acute coronary syndrome with diabetes. Guangdong Medical Journal 2015;13:1257-60.

4 Chen WQ. Analysis of myocardial protective effect of ticagrelor in patients with ST segment elevation acute coronary syndrome with diabetes. Department of Internal Medicine - Cardiovascular 2015;13:04

5 FY X, Zhang BJ, Zhao WL. Effect of ticagrelor on arterial inflammatory reaction and cardiac function in patients with acute coronary syndrome. Department of Cardiovascular Medicine 2016;19:73-7.

6 Jin P, Li L, XR H, et al. Comparative analysis of effects of ticagrelor and clopidogrel on platelet function, inflammatory factors and long-term prognosis in patients with acute coronary syndrome after $\mathrm{PCl}$. Department of Internal Medicine- Cardiovascular 2017;13:203-6.

7 Hao LX, Zhu GH, Cheng S, et al. Comparative study for effectiveness and safety in acute myocardial infarction patients undergoing $\mathrm{PCl}$ between ticagrelor and clopidogrel. PJCCPVD 2017:8:50-3.

8 Moher D, Liberati A, Tetzlaff J, et al. Preferred reporting items for systematic reviews and meta-analyses: the PRISMA statement. BMJ 2009;339:b2535.

9 Choi K-N, Jin H-Y, Shin H-C, et al. Comparison of the antiplatelet effects of once and twice daily low-dose ticagrelor and clopidogrel after percutaneous coronary intervention. Am J Cardiol 2017;120:201-6.

10 Wallentin L, Becker RC, Budaj A, et al. Ticagrelor versus clopidogrel in patients with acute coronary syndromes. N Engl J Med 2009;361:1045-57. 
11 Park D-W, Lee PH, Jang S, et al. Effect of low-dose versus standard-dose ticagrelor and clopidogrel on platelet inhibition in acute coronary syndromes. J Am Coll Cardiol 2018;71:1594-5.

12 Alexopoulos D, Despotopoulos S, Xanthopoulou I, et al. Low-dose ticagrelor versus clopidogrel in patients with prior myocardial infarction. J Am Coll Cardiol 2017;70:2091-2.

13 Julian PT, Higgins SG. Cochrane Handbook for systematic reviews of interventions Cochrane library, 2011.

14 Guo LZ, Kim MH, Jin CD, et al. Comparison of pharmacodynamics between low dose ticagrelor and clopidogrel after loading and maintenance doses in healthy Korean subjects. Platelets 2015:26:563-9.

15 Lee YJ, Kim H, Choi J, et al. Evaluation of pharmacokinetic, pharmacodynamic, efficacy, and safety data of low-dose ticagrelor versus standard dose in East Asians: a systematic review. Ther Clin Risk Manag 2018;14:83-93.

16 Li P, Gu Y, Yang Y, et al. Low-dose ticagrelor yields an antiplatelet efficacy similar to that of standard-dose ticagrelor in healthy subjects: an open-label randomized controlled trial. Sci Rep 2016;6:31838.

17 Li H, Guo J, Carlson GF, et al. Pharmacodynamics, pharmacokinetics, and safety of ticagrelor in Chinese patients with stable coronary artery disease. Br J Clin Pharmacol 2016;82:352-61.

18 Rollini F, Franchi F, Standard- FF. Standard- and low-dose ticagrelor after percutaneous coronary intervention. Circulation 2018;138:1301-3.

19 Sun KB. Clinical efficacy of combination therapy with clopidogrel and clopidogrel in patients with ST segment elevation myocardial infarction treated with percutaneous coronary intervention. China Continuing Medical Education 2017;12:181-4.

20 JG H. Clinical efficacy of low-dose ticagrelor on pafients with coronary heart disease after 6 months of PCI. Chinese Journal of Practical Medicine 2018:8:114-7.

21 Liao ZJ, Chen L. The effect of aspirin combined with ticagrelor in the treatment of acute coronary syndrome. Contemporary Medicine 2017;3:108-9.

22 Meng HY, Kou XJ, Zhou G, et al. Effect of low-dose ticagrelor on platelet aggregation rate and hemorrhage in patients with acute coronary syndrome. Chinese Journal of New Drugs 2017;13:67-9.

23 Song M, Yao ZH, Men JL, et al. Efficacy and safety of low-dose ticagrelor in patients at six months after percutaneous coronary intervention. Chinese General Practice 2017;20:1973-7

24 Liu CY, ZF L. Evaluation on effect of ticagrelor combined with aspirin in acute myocardial infarction. China Prac Med 2017;3:105-7.

25 KB L, YJ X, Han QL. Safety and efficacy of low-dose ticagrelor in patients with unstable angina pectoris and non-revascularization. Chinese Journal of New Drugs 2015:82:2480-4.

26 Xue HJ, Shi J, Liu B, et al. Comparison of half- and standard-dose ticagrelor in Chinese patients with NSTE-ACS. Platelets 2016:27:440-5.

27 Brilakis ES, Patel VG, Banerjee $S$, et al. Medical management after coronary stent implantation: a review. JAMA 2013;310:189-98.

28 Galper BZ, Mauri L. Antiplatelet therapy after coronary stenting. Curr Treat Options Cardiovasc Med 2013;15:1-10.http://www.ncbi.nlm.nih.gov/pubmed/23263881

29 Levine GN, Bates ER, Bittl JA, et al. 2016 ACC/AHA guideline focused update on duration of dual antiplatelet therapy in patients with coronary artery disease: a report of the American College of Cardiology/American Heart Association task force on clinical practice guidelines. J Thorac Cardiovasc Surg 2016;152:1243-75.

30 Bae JP, Dobesh PP, Klepser DG, et al. Adherence and dosing frequency of common medications for cardiovascular patients. Am J Manag Care 2012;18:139-46.

31 Thom S, Poulter N, Field J, et al. Effects of a fixed-dose combination strategy on adherence and risk factors in patients with or at high risk of CVD: the UMPIRE randomized clinical trial. JAMA 2013;310:918-29.
32 Wallentin L, Thorsén M, et al. Ticagrelor versus clopidogrel in patients with acute coronary syndromes. N Engl J Med 2009;36:1045.

33 Serebruany VL, Tomek A, Pya Y, et al. Inferiority of ticagrelor in the PHILO trial: play of chance in East Asians or nightmare confirmation of PLATO-USA? Int J Cardiol 2016;215:372-6.

34 Kheiri B, Osman $M$, Abdalla $A$, et al. Ticagrelor versus clopidogrel after fibrinolytic therapy in patients with ST-elevation myocardial infarction: a systematic review and meta-analysis of randomized clinical trials. J Thromb Thrombolysis 2018;46:299-303.

35 Amsterdam EA, Wenger NK, Brindis RG, et al. 2014 AHA/ACC guideline for the management of patients with non-ST-elevation acute coronary syndromes: Executive summary: a report of the American College of Cardiology/American Heart Association task force on practice guidelines. Circulation 2014;130:2354-94.

36 James S, Angiolillo DJ, Cornel JH, et al. Ticagrelor vs. clopidogrel in patients with acute coronary syndromes and diabetes: a substudy from the platelet inhibition and patient outcomes (Plato) trial. Eur Heart J 2010;31:3006-16

37 Jeong Y-H. "East asian paradox": challenge for the current antiplatelet strategy of "one-guideline-fits-all races" in acute coronary syndrome. Curr Cardiol Rep 2014;16:485.

38 Cannon CP, Harrington RA, James $S$, et al. Comparison of ticagrelor with clopidogrel in patients with a planned invasive strategy for acute coronary syndromes (Plato): a randomised double-blind study. Lancet 2010;375:283-93.

39 Steg PG, Harrington RA, Emanuelsson $\mathrm{H}$, et al. Stent thrombosis with ticagrelor versus clopidogrel in patients with acute coronary syndromes: an analysis from the prospective, randomized Plato trial. Circulation 2013;128:1055-65.

40 Gurbel PA, Bliden KP, Butler K, et al. Randomized double-blind assessment of the onset and offset of the antiplatelet effects of ticagrelor versus clopidogrel in patients with stable coronary artery disease: the ONSET/OFFSET study. Circulation 2009;120:2577-85.

41 Bonello L, Laine M, Kipson N, et al. Ticagrelor increases adenosine plasma concentration in patients with an acute coronary syndrome. J Am Coll Cardiol 2014;63:872-7.

42 Kolandaivelu K, Leiden BB, O'Gara PT, et al. Non-adherence to cardiovascular medications. Eur Heart J 2014;35:3267-76.

43 Hiasa Y, Teng R, Emanuelsson H, Pharmacodynamics EH. Pharmacodynamics, pharmacokinetics and safety of ticagrelor in Asian patients with stable coronary artery disease. Cardiovasc Interv Ther 2014;29:324-33.

44 Husted S, Emanuelsson H, Heptinstall S, et al. Pharmacodynamics, pharmacokinetics, and safety of the oral reversible P2Y12 antagonist AZD6140 with aspirin in patients with atherosclerosis: a double-blind comparison to clopidogrel with aspirin. Eur Heart $J$ 2006;27:1038-47.

45 van Giezen JJJ, Sidaway J, Glaves P, et al. Ticagrelor inhibits adenosine uptake in vitro and enhances adenosine-mediated hyperemia responses in a canine model. J Cardiovasc Pharmacol Ther 2012:17:164-72.

\section{Answers}

1. True

2. False

3. True

4. True

5. True 